\title{
Oscillations of a statically indeterminate system with a finite number of degrees of freedom (the experience with the application of mathematical packages in the technical university course of mechanics)
}

\author{
Alexander Kuznechikov ${ }^{1}$, Dmitry Levitskiy ${ }^{1,\left[{ }^{*}\right]}$, and Sergey Zotov ${ }^{1}$ \\ ${ }^{1}$ Department of the Theoretical Mechanics, Gubkin Russian State University of Oil and Gas (National \\ Research University), 65 Leninsky Prospekt, Moscow, 119991, Russia
}

\begin{abstract}
Symbolic mathematics packages give the opportunity to execute the difficult symbolic transformations with use of computer, abandoning graphic methods. The resilient weightless beam fixed by resilient links and carrying two concentrated masses is considered. Instead of building the bending moment diagram and the later use of Vereshchagin's method for disclosure of static indeterminacy, the equation of distribution of the bending and single moments along the beam length is written, and Mohr's integral is calculated.
\end{abstract}

\section{Introduction}

Students have to create and perform operations on the inertia and flexibility matrices, when studying oscillations of systems with a finite number of degrees of freedom. If a discrete system is statically indeterminate (such problems can occur when studying structural mechanics), then the solution of the problem becomes cumbersome. In addition to calculating the potential energy, it is necessary to disclose static indeterminacy, to calculate the constraint force, which is traditionally performed using graph-analytical methods. Integration of symbolic mathematics packages into the educational process changes things dramatically. The students get the opportunity to perform difficult symbolic transformations with use of the computer, abandoning the graphical methods.

When solving this problem, the convenience of the symbolic mathematics package DERIVE [1] which was one of the five best and widely distributed packages for a long time, is demonstrated.

The purpose of this article is thus demonstration of advantages of applying symbolic mathematics packages while performing tasks of the systems oscillation theory with finite number of degrees of freedom. This is referred to the practical experience of such implementation in the technical university course of mechanics.

\footnotetext{
* Corresponding author: levitskiy.d@gubkin.ru
} 


\section{Practical example}

As an example, the resilient weightless beam fixed by resilient links and carrying two concentrated masses is considered. Instead of building the bending moment diagram and the later use of Vereshchagin's method for disclosure of static indeterminacy, the equation of distribution of the bending and single moments along the beam length is written, and Mohr's integral is calculated.

Calculation of potential energy and creation of a matrix is a cumbersome and an exigent task in case of the "manual" computation, but it is carried out by means of the mathematical package without effort.

We now need to determine the base frequency and a form of oscillations [2] of the resilient mechanical structure represented in Figure 1 under the following mechanical and geometric properties:

$m_{m}=1.5 \cdot 10^{4} \mathrm{~kg}$ - mass of the drilling platform's mast;

$m_{k}=10^{4} \mathrm{~kg}-$ mass of the upper structure;

$E I=4 \cdot 10^{4} \mathrm{Mn} \cdot \mathrm{m}^{2}-$ flexural rigidity of the mast;

$c_{1}=100 \mathrm{Mn} / \mathrm{m}$ - equivalent linear rigidity of the resilient half-space around the base;

$c_{2}=150 \mathrm{Mn} / \mathrm{m}-$ equivalent rigidity of cables;

$c_{\mathrm{c}}=2000 \mathrm{Mn} / \mathrm{m}$ - equivalent spiral rigidity of the resilient half-space around the base;

$h=90 \mathrm{~m}-$ mast height;

$l=60 \mathrm{~m}-$ distance between fixing points of the equivalent springs.

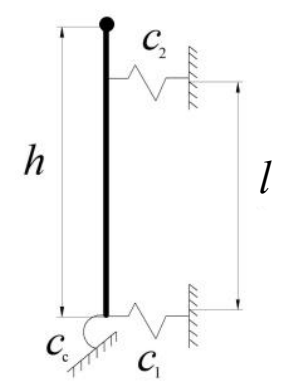

Figure 1. A resilient mechanical structure.

Let us concentrate the mass of the whole beam in beam middle. The resilient massive beam will turn into a system with two degrees of freedom (Figure 2).

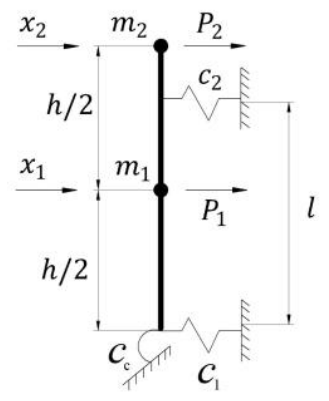

Figure 2. System with two degrees of freedom.

The position of system is characterized by the generalized coordinates vector with components $x_{1}, x_{2}$. As this takes place the mass of points is respectively equal to $m_{1}=$ $m_{m}, m_{2}=m_{k}$. Kinetic energy of this system is: 


$$
E=0.5\left(m_{1} \dot{x}_{1}^{2}+m_{2} \dot{x}_{2}^{2}\right)
$$

Introducing a vector of the generalized velocities, it is possible to write down kinetic energy in a matrix form:

$$
E=0.5 \dot{\overrightarrow{\boldsymbol{x}}}^{T} \cdot \boldsymbol{M} \dot{\overrightarrow{\boldsymbol{x}}}
$$

where is the inertia matrix $\boldsymbol{M}$ contains nonzero elements only on the main diagonal and has the form:

$$
\boldsymbol{M}=\left[\begin{array}{cc}
m_{1} & 0 \\
0 & m_{2}
\end{array}\right]=10^{4}\left[\begin{array}{cc}
1.5 & 0 \\
0 & 1
\end{array}\right]
$$

Let us calculate the potential energy in the resilient system, expressing it through the generalized forces of inertia of $P_{1}$ and $P_{2}$.

$$
V=V_{s}+V_{b}=\int_{0}^{h[M(y)]^{2} d y} \frac{F_{B}^{2}}{2 E I}+\frac{F_{D}^{2}}{2 c_{1}}+\frac{M_{D}^{2}}{2 c_{c}}
$$

$V_{s}$ - potential energy of resilient supports which the rigid beam (Figure 3) to is fixed;

$V_{b}$ - potential energy of the resilient beam having the rigid supports (Figure 4);

$E I$ - flexural rigidity of the beam;

$M(y)$ - bending moment depending from $y$;

$F_{B}$ - reaction in the point $B$;

$F_{D}, M_{D}$ - reactions in the point $D$.

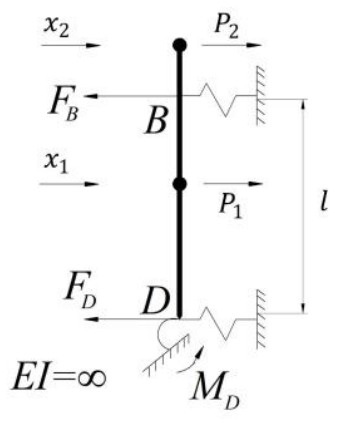

Figure 3. Potential energy of resilient supports which fix the resilient beam.

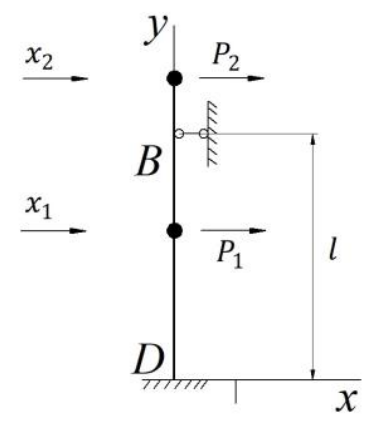

Figure 4. Potential energy of the resilient beam with the rigid support. 
For totality of planar system of parallel forces the structure is once statically indeterminate.

Let us consider two cases:

1) the generalized force is applied above $\operatorname{support} B$,

2) the generalized force is applied below support $B$.

We will choose the main system, having rejected support $B$ (Figure 5).

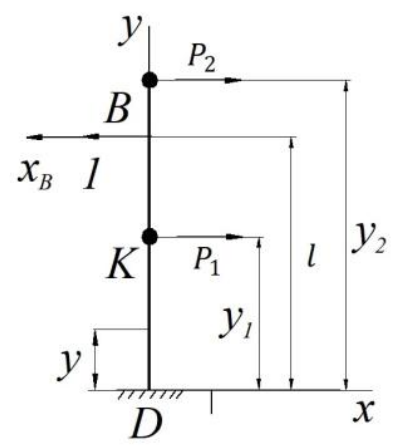

Figure 5. Main system with rejected support B.

For calculation of reaction in point $B$ depending from force $P_{2}$ we write down Mohr's integral for point $B$ depending from forces $X_{B}$ and $P_{2}$.

$$
\delta_{B}=\int_{0}^{l} \frac{M^{0} M\left(P_{2}\right) d y}{E I}+\int_{0}^{l} \frac{M^{0} M\left(X_{B}\right) d y}{E I}=0
$$

where $M^{0}$ is the bending moment from unit force.

In a local system of coordinates XDY, we will find the equation of the bending moment diagram $M\left(X_{B}\right)$ depending from force $X_{B}$ arising in section $K$. For this purpose we will write down expression of the moment from force $X_{B}$ relating to the point $K$ with coordinates $(0, y, 0)$ in an analytical form: for $y<l$

$$
\overrightarrow{\boldsymbol{M}}\left(\vec{X}_{B}\right)=-\left|\begin{array}{ccc}
\overrightarrow{\boldsymbol{\imath}} & \overrightarrow{\boldsymbol{\jmath}} & \overrightarrow{\boldsymbol{k}} \\
\mathbf{0} & (\boldsymbol{l}-\boldsymbol{y}) & \mathbf{0} \\
-\boldsymbol{X}_{B} & \mathbf{0} & \mathbf{0}
\end{array}\right|=-\overrightarrow{\boldsymbol{k}}(l-y) X_{B}
$$

or $M\left(X_{B}\right)=(y-l) X_{B}, y<l$.

The minus sign is taken because $M\left(X_{B}\right)+M_{K}\left(X_{B}\right)=0$, where $M_{K}\left(X_{B}\right)$ is moment from force $X_{B}$ relating to the point $K$.

Let us similarly find $M\left(P_{2}\right)$.

$$
\overrightarrow{\boldsymbol{M}}\left(\overrightarrow{\boldsymbol{P}}_{2}\right)=-\left|\begin{array}{ccc}
\overrightarrow{\boldsymbol{\imath}} & \overrightarrow{\boldsymbol{\jmath}} & \overrightarrow{\boldsymbol{k}} \\
\mathbf{0} & \left(\boldsymbol{y}_{2}-\boldsymbol{y}\right) & \mathbf{0} \\
\boldsymbol{P}_{2} & \mathbf{0} & \mathbf{0}
\end{array}\right|=\overrightarrow{\boldsymbol{k}}\left(y_{2}-y\right) P_{2}
$$

or $M\left(P_{2}\right)=\left(y_{2}-y\right) P_{2}$.

The bending moment from unit force $M^{0}$ we will obtain by having replaced force $X_{B}$ with unit. Thus $M^{0}=y-l$. The calculated dependences of bending moments on coordinates correspond to building the bending moments diagram of compressed fibres. As a result, Mohr's integral for force lying above support $B$ will take form:

$$
\int_{0}^{l}(y-l) P_{2}\left(y_{2}-y\right) d y+\int_{0}^{l}(y-l)^{2} X_{B} d y
$$

Record of the last formula and calculation of $X_{B}$ reaction by using DERIVE package [4]: 
Here INT is designation of integral,

p sub2 - designation of force $P_{2}$;

y sub2 - designation of $y_{2}$;

$\mathrm{x}$ sub - designation of force $X_{B}$.

$$
\begin{aligned}
& \operatorname{INT}\left((y-1)^{*}\right. \text { p sub2(y sub2-y),y,0,1)+INT((y-1)^2*x subb,y,0,1)=0 } \\
& 0.333333 * 1 \wedge 3 * \mathrm{x} \text { subb-0.166666*1^2*p sub } 2 *(3 * \mathrm{y} \text { sub2-1 })=0 \\
& \mathrm{x} \mathrm{subb}=0.5 * \text { p sub } 2 *(3 * \text { y sub2-1 }) / 1
\end{aligned}
$$

As a result:

$$
X_{B}=\left(0.5 P_{2}\left(3 y_{2}-l\right)\right) / l
$$

Let us similarly calculate value of force $X_{B}$ depending of force $P_{1}$ applied below support $B$. In this case Mohr's integral has form:

$$
\int_{0}^{y_{1}}(y-l) P_{1}\left(y_{1}-y\right) d y+\int_{0}^{l}(y-l)^{2} X_{B} d y=0
$$

After calculations we will obtain:

$$
X_{B}=\left(0.5 P_{1} y_{1}^{2}\left(3 l-y_{1}\right)\right) / l^{3}
$$

Reaction in support $B$ depending on the generalized inertial forces can be calculated in accordance with the formula:

$$
\begin{aligned}
& F_{B}=\left(0.5 P_{2}\left(3 y_{2}-l\right)\right) / l+\left(0.5 P_{1} y_{1}^{2}\left(3 l-y_{1}\right)\right) / l^{3}= \\
& \quad=\left(0.5 P_{2}(3 h-l)\right) / l+\left(0.5 P_{1} h^{2}(6 l-h)\right) /\left(8 l^{3}\right)
\end{aligned}
$$

For calculations of potential energy of the deformed springs we will find constraint reactions in the point $D$ depending of forces $P_{1}$ and $P_{2}$. Writing down and solving equilibrium equations of planar system of parallel forces, we will find

$$
\begin{gathered}
F_{D}=P_{1}+P_{2}-F_{B}=1.5 P_{2}(1-h) / l+P_{1}\left(8 l^{3}-3 l h^{2}+0.5 h^{3}\right) /\left(8 l^{3}\right) \\
M_{D}=P_{1} h / 2+P_{2} h-X_{B} l=P_{1} h / 2+0.5 P_{2}(l-h)-P_{1} h^{2}(6 l-h) /\left(16 l^{2}\right)
\end{gathered}
$$

Expression of bending moment in arbitrary cross-section of the beam can be written down, using the Heaviside function $\sigma(l-y)=1$, for $l-y>0, \sigma(l-y)=0$, for $l-y<0$.

$$
M(y)=P_{1}(h-y)-F_{B}(l-y) \cdot \sigma(l-y)+P_{1}(0.5 h-y) \cdot \sigma(0.5 h-y)
$$

Let us calculate potential energy of the resilient beam for the preset values of $h$ and $l$ :

$$
V_{b}=2.2\left(5120 P_{2}^{2}-1728 P_{1} P_{2}+351 P_{1}\right) /(E I)
$$

Afterwards values of variables $h$ and $l$ are defined, potential energy of resilient beam is written down and calculation of the beam is made. For further calculations by using DERIVE package it is necessary to change from index variables to regular ones. This is due to restricted opportunities of DERIVE package during the work with index variables. Therefore, the designations $P_{2}=p, P_{1}=q$ will be used in files. As a result by using DERIVE package: 


$$
V=2.012 \cdot 10^{-7}\left(1.74 p^{2}+0.39 p q+0.74 q^{2}\right)
$$

Further the matrix of influence coefficients is formed

$$
\alpha=2.012 \cdot 10^{-7}\left|\begin{array}{cc}
1.74 & 0.195 \\
0.195 & 0.74
\end{array}\right|
$$

We find proper frequencies of system as matrix eigenvalues $[\alpha][\mathrm{M}]$. The least proper frequency is equal to $13.72 \mathrm{~s}^{-1}$.

For comparison we will define proper frequencies for similar statically determinate system, without support $B$. Reactions of resilient links are equal:

$$
\begin{gathered}
M_{D}=P_{1} h / 2+P_{2} h \\
M(y)=P_{2}(h-y)+P_{1}(0.5 h-y) \cdot \sigma(0.5 h-y)
\end{gathered}
$$

Further, we will calculate potential energy of resilient system by the formulas given above. After potential energy calculation and items grouping we will receive:

$$
V=2.03 \cdot 10^{-6}\left(2.50 P_{1}^{2}+0.44 P_{2}^{2}+1.94 P_{1} P_{2}\right)
$$

Proper frequencies of oscillations in such system, are equal to $3.45 \mathrm{~s}^{-1}$ and $29.17 \mathrm{~s}^{-1}$, respectively. The least proper frequency has decreased that is explained by lowering the system rigidity.

\section{Conclusions}

In this paper we demonstrated on one typical example our positive experience in implementing symbolic mathematics packages in teaching courses of mechanics and similar at our technical university, which allows us to modernize our teaching methods.

\section{References}

[1] I. M. Babakov, The theory of Oscilations (in Russian) (Moscow Drofa 2004)

[2] L. Magiera, General Physics Problem Solving With Cas Derive (Nova Science Pub Inc 2001)

[3] W.F. Smith, Waves and Oscillations: A Prelude to Quantum Mechanics (Oxford University Press 2010)

[4] V.P. Dyakonov Computer Derive Systems Derive: Self-Tutorial and Guidance the user. (in Russian) (Moscow SOLON-R 2002) 